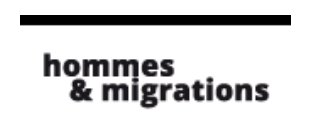

Hommes \& migrations

Revue française de référence sur les dynamiques

migratoires

$1292 \mid 2011$

La discrimination au féminin pluriel

\title{
Un réseau d'acteur contre les discriminations
}

Entretien avec Ahmed Serraj, directeur de l'association Boulevard des Potes à Bordeaux

\section{Marie Poinsot}

\section{(2) OpenEdition \\ Journals}

\section{Édition électronique}

URL : http://journals.openedition.org/hommesmigrations/639

DOI : 10.4000/hommesmigrations.639

ISSN : 2262-3353

\section{Éditeur}

Musée national de l'histoire de l'immigration

\section{Édition imprimée}

Date de publication : 1 juillet 2011

Pagination : 114-119

ISSN : 1142-852X

Référence électronique

Marie Poinsot, «Un réseau d'acteur contre les discriminations », Hommes \& migrations [En ligne], 1292 | 2011, mis en ligne le 31 décembre 2013, consulté le 19 avril 2019. URL : http://

journals.openedition.org/hommesmigrations/639; DOI : 10.4000/hommesmigrations.639

Tous droits réservés 


\section{Un réseau d’acteur contre les discriminations} Entretien avec Ahmed Serraj,
directeur de l'association Boulevard des Potes
à Bordeaux

Réalisé par Marie Poinsot

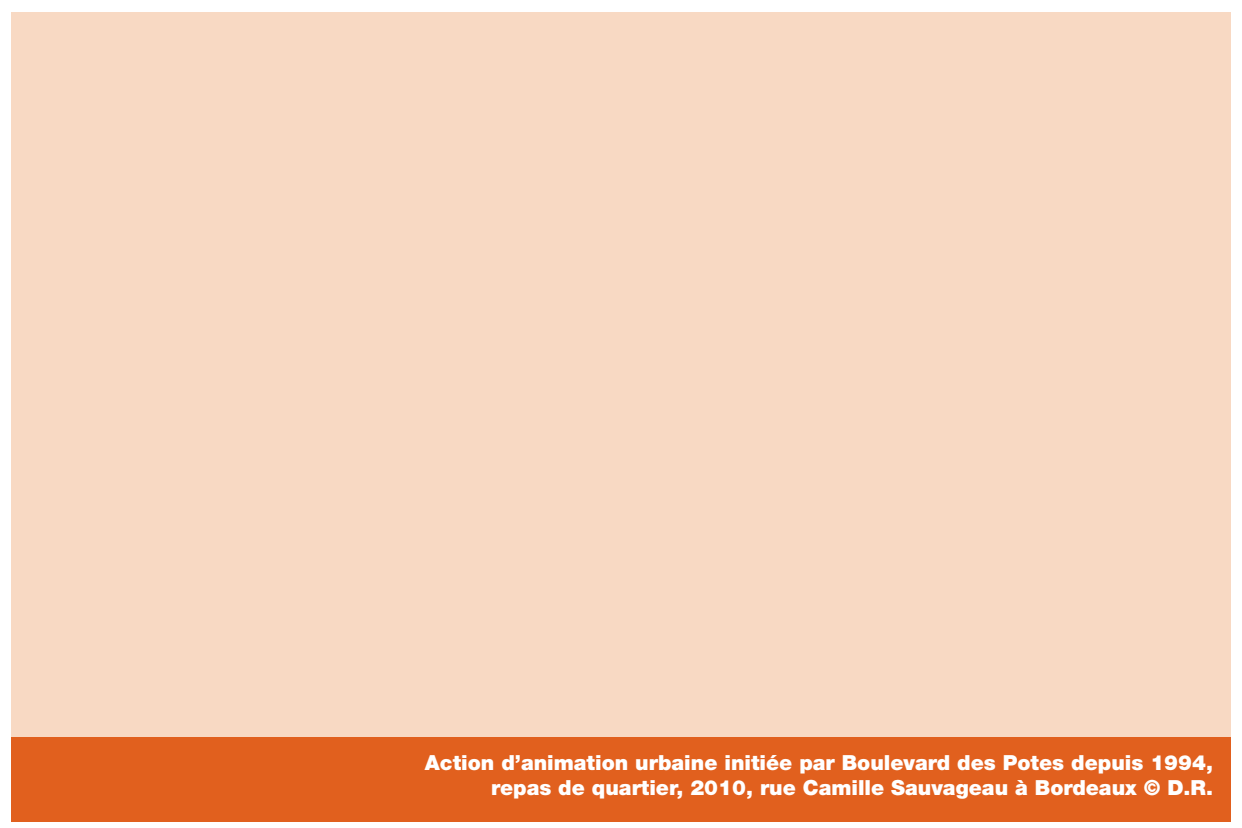

Association agréée d'“éducation populaire", Boulevard des Potes

a été créé en 1991 à l'initiative de militants associatifs, de travailleurs sociaux et d'universitaires.Cette association a pour objet de promouvoir

toute initiative qui revitalise le tissu social, de renforcer la solidarité et d'encourager les actions qui participent à la démocratie et à la lutte contre les discriminations. Ces engagements sont portés

par des initiatives permanentes de sensibilisation de la jeunesse, de formation des acteurs professionnels, d'appui aux militants et acteurs associatifs, de permanences juridiques concernant le droit des étrangers, d'animation urbaine. 


\section{Hommes \& Migrations : À quel moment et pourquoi votre association a-t-elle décidé d'intervenir sur les discriminations envers les femmes d’origine étrangère ?}

Ahmed Serraj : Boulevard des Potes est une association d'éducation populaire qui agit dans le champ de la lutte contre le racisme et les discriminations, et cela depuis sa fondation il y a vingt ans. La question de la lutte contre les discriminations est donc inhérente à sa création, à ses missions, à ses convictions. Elle intervient en réseau avec d'autres associations ceuvrant dans le domaine de l'anti-racisme qui sont apparues au début des années quatre-vingt. La mobilisation en faveur de l'égalité et de la lutte contre les discriminations qui touchent les femmes d'“origine étrangère" (si cette notion a un sens quand les personnes sont nées en France et y vivent depuis vingt-cinq à cinquante ans) découle de cet investissement initial.

Pour autant, le sujet est relativement récent dans l'histoire des luttes pour l'égalité des droits ou celle des combats féministes. Un certain nombre d'associations se sont associées à cette question sans nécessairement avoir les compétences pour accompagner sérieusement et utilement les victimes de discriminations. Les acteurs des secteurs public et privé de l'emploi, de l'éducation, des loisirs, des sports s'avèrent également démunis pour comprendre les phénomènes et intervenir. C'est pourquoi nous nous engageons sur ce terrain-là et avons développé des actions d'accompagnement en Gironde et en Aquitaine.

Ce sujet est au cceur de notre intervention quotidienne; c'est un axe central qui se déploie sur des activités multiples en termes d'accueil, d'accompagnement, de soutien aux victimes, de sensibilisation et de prévention, ou de formation et d'éducation à la lutte contre le racisme et les discriminations. En direction des publics jeunes et moins jeunes. Par exemple, dans le domaine de l'animation, Boulevard des Potes conduit un brevet d'État de développement social et participation citoyenne sous l'égide de la Direction régionale de la jeunesse des sports et de la cohésion sociale (DRJSCS Aquitaine).

Les publics féminins d'origine étrangère ne peuvent retrouver confiance en la loi et les institutions qu'en étant reconnus comme des citoyennes à part entière. Mais cela demande un accompagnement personnalisé et du temps. Ces femmes ont du mal à déposer une plainte car elles se sentent stigmatisées ou humiliées.

Il y a donc un effort particulier d'information et de sensibilisation à faire en direction des services publics, des acteurs privés et bien sûr de ces femmes. Il faut analyser leur capacité à réagir. Entre la prise de conscience de leur propre situation et le moment où elles font une démarche, il y a un temps qu'il faut comprendre et prendre en compte. 


\section{H\&M : Quel diagnostic votre association fait-elle sur l'ampleur et la nature des discriminations ? Comment déterminez-vous les réalités vécues par les femmes étrangères en termes de discriminations ?}

A. S. : La lutte contre les discriminations était difficilement reconnue comme un enjeu important puisque les institutions intégraient souvent les inégalités de traitement en direction des femmes d'origine étrangère comme une situation de fait. Depuis la loi du 16 novembre 2001, on assiste à l'émergence et à la visibilité de cette question, activée par l'outil juridique. La loi a mis au jour les réalités des discriminations restées cachées jusqu'à cette date. Par exemple, l'état de grossesse n'était pas envisagé comme une situation de discrimination, ni la maternité comme un frein à la promotion professionnelle ou au recrutement sur le marché du travail. C'est devenu un 18 critère, en complément de celui du genre, qui révèle des situations que seule la loi a permis d'identifier à partir de sa mise en pratique.

De la même manière, les discriminations multifactorielles constituent un domaine très peu abordé par les travaux de recherche. Pourtant, ce double critère, qui croise le genre et l'origine, touche tous les domaines de l'éducation, de l'animation, de la formation et de l'emploi. Il existe des métiers complètement sexués. Certains secteurs recrutent en fonction de représentations stéréotypées. Par exemple, la bijouterie embauche essentiellement des jeunes femmes d'apparence européenne qui "présentent bien". Dans certains lycées professionnels, au contraire, il n'y a que des garçons ou que des filles. Ce n'est que récemment que notre association a pris conscience de l'effet cumulé des critères de discrimination en travaillant concrètement sur les situations des publics accueillis et en intégrant des compétences supplémentaires sur la problématique de l'égalité de traitement. Un des enjeux sur lesquels il faut développer des énergies décuplées, c'est la formation des milieux professionnels.

Le monde de l'éducation et de l'apprentissage, par exemple, tout comme le reste de la société, n'est pas exempt du phénomène de discrimination. Dans l'éducation et l'apprentissage, les systèmes de représentations et de préjugés peuvent induire des discriminations indirectes, inconscientes et diffuses. Les acteurs concernés, en particulier les formateurs de Centre de formation des apprentis (CFA), chef d'établissement, conseiller principal d'éducation (CPE), enseignants, se trouvent confrontés à des situations discriminatoires sur lesquelles ils ont peu de prise, ce qui peut entraîner chez eux un sentiment d'impuissance et de désarroi. Ils ont besoin de disposer d'outils et de méthodes pour prévenir les discriminations, agir contre elles, et restaurer l'égalité de traitement dans l'accueil, l'orientation, la notation, la sanction et l'accès aux stages. 


\section{H\&M : Comment les discriminations multifactorielles affectent-elles d'autres secteurs que le marché du travail ?}

A. S. : Un des éléments qui ressort, c'est l'accès au logement pour les femmes étrangères mais aussi pour les familles monoparentales, composées de femmes souvent en situation sociale et économique précaire, élevant seules leurs enfants et venant d'un quartier stigmatisé. Elles rencontrent de grandes difficultés pour accéder à un logement dans le parc public ou privé, dans une zone urbaine... Elles se trouvent souvent reléguées dans le péri-urbain, à l'extérieur des agglomérations. On peut parler de domaine multifactoriel quand on propose un logement éloigné des activités économiques de l'emploi, qui aggrave leur situation de travail. C'est un phénomène qui a pris de l'importance ces dernières années, avec des représentations négatives de ces femmes dans les agences, chez les propriétaires ou les organismes HLM.

Par ailleurs, dans le monde du sport, malgré les grands principes et valeurs qu'il porte, les pratiques sont sexuées, y compris à un haut niveau de compétition, puisque les femmes ne sont ni couvertes par les médias ni primées de la même manière que les hommes. Dans les instances dirigeantes du sport, les femmes ont très peu de responsabilités, quelles que soient les échelles territoriales. Les représentations sont organisées indirectement de manière très discriminante. Il faut faire remonter ces situations de discrimination auprès des comités olympiques, des fédérations régionales ou des associations sportives afin qu'elles puissent être prises en compte dans une logique d'égalité de traitement.

\section{H\&M : Quelle est l'évolution de vos actions dans le temps, avec ses freins et ses limites?}

A. S. : Si le niveau de prise de conscience des discriminations progresse et si le sujet de l'égalité des droits et de traitement relève d'un engagement citoyen dans la sphère publique, pour autant on constate que dans la délivrance de services aux publics, les agents continuent à discriminer parfois de manière indirecte ou inconsciente. C'est pour cela que notre association les incite à acquérir des compétences spécifiques en déconstruisant leurs représentations et les idées qu'ils ont sur les publics qu'ils côtoient, notamment sur les femmes d'origine étrangère. Cette internalisation de la lutte contre les discriminations dans tous les services publics prend du temps. Des formations sont organisées pour transférer tout l'outillage juridique et méthodologique, en vue de modifier les pratiques professionnelles tout en préservant les énergies sur les territoires où ces agents interviennent afin que ce thème soit toujours présent dans leurs activités. Un sujet d'ancrage, croisant volonté politique et publique sur le territoire. 
Nous proposons des formations en interne qui évoluent en fonction de l'actualité et des mutations de ce thème. À la demande de François Dubet et de son équipe de l'université Bordeaux-Segalen, Boulevard des Potes a participé à des séances de réflexion avec les chercheurs et les acteurs qui travaillent sur la lutte contre les discriminations : Comment ces acteurs influent-ils sur les enjeux de société ? Nous participons généralement à des démarches d'action recherche pour mêler savoirs et savoir-faire. À ce titre, nous avons constitué un comité scientifique présidé par Abdou Tahari-Chaoui ${ }^{(1)}$ et auquel participent Isabelle Daugareilh, juriste spécialiste du droit social des étrangers et de la lutte contre les discriminations ${ }^{(2)}$, et Rachid Alaoui ${ }^{(3)}$, socioéconomiste, universitaire et expert des questions de lutte contre les discriminations.

\section{H\&M : Quelles sont vos relations avec les entreprises ?}

A. S.: Elles sont conditionnées par un point d'accroche possible dans la mesure où une association n'est pas un syndicat, ni l'Inspection du travail ou le Conseil régional. La légitimité n'est pas facile à acquérir auprès du monde du travail. Il y a beaucoup à faire dans ce secteur, mais notre association n'y est pas, encore, considérée comme un interlocuteur à part entière. Un partenariat s'est mis en place avec la chambre de l'artisanat et des métiers. Le projet consistait à s'appuyer sur la lutte contre les discriminations comme levier à la pérennisation des petites et moyennes entreprises dont les artisans partent à la retraite sans relève assurée. Cette expérimentation a été réalisée dans les métiers de la coiffure, de la boulangerie, de la pâtisserie et de la boucherie, notamment à travers l'accès aux stages des jeunes apprentis. On a édité des plaquettes, des affiches, sans les désigner ou les stigmatiser. C'est un travail de longue haleine qui va au-delà des campagnes d'information.

Les actions en justice sont portées par SOS-Racisme quand il s'agit d'un refus d'emploi ou de discriminations dans les relations de travail car nos énergies associatives sont complémentaires et cohérentes. Il faut savoir structurer un collectif d'associations sur la région car la lutte contre les discriminations se construit avec l'ensemble des acteurs du territoire (État, police, justice, collectivités territoriales, élus, services publics, associations, entreprises, acteurs de l'emploi, acteurs du logement, etc.).

\section{H\&M : La crise économique aggrave-t-elle la situation des femmes d'origine étrangère?}

A. S. : Parmi les publics les plus fragiles et sensibles, les femmes subissent les conséquences de la crise économique de manière plus violente. On est confronté à des situations complexes et nombreuses, mais je ne peux pas vous proposer une 
analyse plus fine des effets de la crise sur les situations de discrimination. Lobjectif est de maintenir cet enjeu dans des lieux institutionnels qui peuvent en faire le portage le plus efficace possible auprès des publics menacés. La question de l'origine sociale devrait être prise en compte comme critère de discrimination caché ou aveugle. Quand les gens habitent des quartiers dits "sensibles", avec des minima sociaux, quel que soit leur genre, ils subissent des discriminations supplémentaires en vertu de leur statut social. Il faut également considérer les situations d'exclusion comme des vecteurs et facteurs de discriminations car les personnes précaires sont également victimes de représentations négatives comme en inspirent l'origine ou le genre. Cette situation sociale des personnes exclues ne recoupe pas forcément des appartenances culturelles, religieuses ou des origines étrangères. La ville de La Courneuve, par exemple, a porté plainte contre un organisme en raison de discriminations fondées sur le lieu de résidence. Ce serait un $19^{\mathrm{e}}$ critère reconnu de discrimination pour lequel nous militons.

1. Président du Boulevard des Potes et directeur d'une association de prévention spécialisée et de travail social.

2. Directrice de recherche au CNRS et membre du Centre de droit comparé du travail et de la Sécurité sociale (COMPTRASEC), université Montesquieu Bordeaux-IV.

3. Chargé de cours à l'université de Paris-IV Sorbonne et à l'université de Reims. 